\title{
Use and Duration of Chemotherapy in Patients With Metastatic Breast Cancer According to Tumor Subtype and Line of Therapy
}

\author{
Davinia S.E. Seah, MD, MPHª Ines Vaz Luis, MDa; Erin Macrae, $\mathrm{MD}^{\mathrm{b}}$; Jessica Sohla; \\ Georgia Litsas, MSN, RN, NPa; Eric P. Winer, MDa; Nancy U. Lin, MDª and Harold J. Burstein, MD, PhD
}

\begin{abstract}
Benefits of chemotherapy vary in patients with metastatic breast cancer (MBC). This article describes the impact of tumor subtype and the line of therapy on the duration of chemotherapy. Clinicopathologic characteristics were extracted from the medical records of 199 consecutive patients with MBC at Dana-Farber Cancer Institute and analyzed according to subtype. Tumor subtypes were classified as hormone receptor (HR)-positive, triple-negative (TNBC), or HER2-amplified breast cancer. Duration of chemotherapy of each line was defined as the start of a chemotherapy regimen to the start of the next line of therapy as a result of progression or toxicity. There were 96,44 , and 59 patients with $\mathrm{HR}^{+}$, TNBC, and HER2amplified breast cancer, respectively. Median age at MBC diagnosis was 53 years. Median overall survivals were 32 and 54 months for HER2-amplified disease, 36 months for $\mathrm{HR}^{+}$breast cancer, and 17 months for TNBC $(P<.0001)$. Patients with HER2-amplified disease received the most lines (median, $4 ; P=.032$ ) and the longest duration of chemotherapy for every line. The median duration of chemotherapy in HER2-amplified patients remained at more than 4 months even out to sixth-line therapy. Patients with TNBC tended to receive the shortest duration of chemotherapy for every line of therapy. Tumor subtypes influence the number of lines, duration of chemotherapy, and survival. Among patients with $\mathrm{HR}^{+}$and HER2-amplified disease who undergo chemotherapy beyond the third line, substantial rates of prolonged therapies suggest clinical benefit. The role of advanced (greater than third) chemotherapy lines in improving survival of all patients with $\mathrm{MBC}$ warrants further study. (J Natl Compr Canc Netw 2014;12:71-80)
\end{abstract}

From the a Department of Medical Oncology, Dana-Farber Cancer Institute, Boston, Massachusetts, and ${ }^{b}$ Department of Medical Oncology, The Ohio State University College of Medicine, Columbus, Ohio.

Submitted February 15, 2013; accepted for publication July 10, 2013.

This work was supported by the Metastatic Research Fund (anonymous donor to Drs. Winer and Lin), Megan Lally Memorial Fund, and Susan G. Komen for the Cure (Dr. Winer). Dr. Lin has disclosed that she received research funding from Genentech, Inc., GlaxoSmithKline, and Novartis. The remaining authors have disclosed that they have no financial interests, arrangements, affiliations, or commercial interests with the manufacturers of any products discussed in this article or their competitors. Correspondence: Harold J. Burstein, MD, PhD, Department of Medical Oncology, Division of Women's Cancers, Dana-Farber Cancer Institute, 450 Brookline Avenue, Boston, MA 02215. E-mail: hal_burstein@dfci.harvard.edu

\section{Background}

Advances in breast cancer treatment have significantly improved survival for patients with metastatic breast cancer (MBC) from 1 to 2 years in the 1970s to 1 to 3 years today. ${ }^{1-3}$ However, MBC remains incurable, and the goals of treatment are prolongation of survival and palliation of symptoms. ${ }^{4,5}$

Although gene profiling studies have advanced the understanding of the heterogeneity of breast cancer, ${ }^{6,7}$ most clinicians broadly classify breast cancer into 3 subtypes: hormone receptor (HR)-positive, HER2amplified, and triple-negative breast cancer (TNBC)..$^{8-11}$ TNBC is characterized by a younger patient distribution and a higher mortality rate compared with other subtypes. ${ }^{9} 10$ HER2 amplification historically conferred a worse prognosis, ${ }^{12}$ but this has improved with the introduction of HER2-targeted therapy. ${ }^{13} \mathrm{HR}^{+}$breast cancers have traditionally been associated with the best prognosis but are characterized by an ongoing risk of late relapse relative to the other subtypes. ${ }^{14}$

Decisions about treatment are increasingly being tailored to individual patient characteristics, such as tumor subtype. This clinical classification identifies targets with established data on treatment efficacy; hormonal therapies for $\mathrm{HR}^{+}$breast cancer; and HER2directed therapies for HER2-amplified breast cancer. ${ }^{15,16}$ However, despite the heterogeneity of breast cancer, almost all patients with $\mathrm{MBC}$ will receive chemotherapy at some point. Compared with patients who have other solid tumors, patients with MBC have a larger number of therapeutic options available. ${ }^{17}$ The likelihood of response and prolonged tumor control is highest with the first line of chemotherapy, with limited data on the efficacy of later lines of chemotherapy despite its very common use in clinical practice. ${ }^{18-20}$ Few data also ex- 
ist regarding the impact of tumor subtype on the likelihood of benefit with later lines of treatment. Although some data exist from prospective clinical trials conducted in patients with refractory $\mathrm{MBC}$, there are unavoidable limitations in basing estimates of the benefit of chemotherapy or survival solely on these trials, given that eligibility and exclusion criteria limit the types of patients who enter the trials, and may not be representative of patients with MBC seen in everyday practice.

Patients with advanced cancer want information about their prognosis and what to expect from their disease and treatments. ${ }^{21,22}$ A better sense of the likelihood of clinical benefit, survival probabilities, and prognostic factors could inform decisions on whether to embark on another line of palliative chemotherapy. ${ }^{23}$ This retrospective study describes the pattern of palliative chemotherapy use according to tumor subtypes in patients treated for MBC between 2004 and 2007, and the duration of chemotherapy as defined by the time from the initiation of one chemotherapy regimen to the initiation of the next line of therapy as a result of disease progression or toxicity.

\section{Methods}

The medical records of 318 consecutive patients diagnosed with MBC between January 1, 2004, and December 31, 2007, and treated at the DanaFarber Cancer Institute (DFCI) were reviewed. The end date of 2007 was chosen to ensure at least 4.5 years of potential follow-up time. Patient characteristics, histology, treatment history, and survival data were abstracted from electronic medical records. Patients were excluded if the tumor subtype was unknown, they did not have distant metastases or locally advanced disease treated with palliative intent, they did not receive any palliative chemotherapy, they did not receive at least one line of palliative chemotherapy at DFCI, they had another active malignancy, or they had more than one breast primary.

\section{Definitions}

Breast cancer subtypes were classified as $\mathrm{HR}^{+}$(estrogen receptor $[E R]$-positive or progesterone receptor [PR]-positive and HER2 nonamplified), TNBC (HR-, HER2 nonamplified), or HER2-amplified (immunohistochemistry score of $3+$, fluorescence in situ hybridization amplification $\geq 2.0$, or both; $\mathrm{HR}^{+}$, or $\mathrm{HR}^{-}$). ER and PR status were classified as abstracted from pathology reports according to the classification applicable at diagnosis.

The duration of chemotherapy of each line was defined as the initiation of one chemotherapy regimen to the initiation of the next line of therapy as a result of disease progression or toxicity. The term chemotherapy may refer to traditional chemotherapy or a clinical trial therapy. Endocrine monotherapy and vaccine trials were excluded. Details on what constituted a line of chemotherapy and how the duration was calculated is outlined in Appendix A (available online, in this article, at JNCCN.org).

This study was approved and performed according to the guidelines of the DFCI Institutional Review Board.

\section{Statistical Analysis}

Descriptive statistics were used to summarize patients' demographic and clinical characteristics. Fisher exact tests were used to examine categorical variables and Kruskal-Wallis tests were used to examine continuous variables between the subtypes. All $P$ values presented are 2 -sided; a $P$ value of less than 0.05 was considered significant.

Overall survival and duration of chemotherapy were calculated using the Kaplan-Meier method. Overall survival time was defined as the time between metastatic diagnosis and death. Death was considered an event. If the patient was still alive at the time of medical record review or was lost to follow-up, overall survival was censored at the date of last entry in the medical record on which the patient was still alive. To determine the duration of chemotherapy, progression and toxicity were considered events. The line of therapy was censored if the patients were undergoing ongoing therapy at the time of medical record review; they were lost to followup; or did not experience progression on therapy. $\mathrm{Pa}-$ tients still on treatment at time of last follow-up or who were lost to follow-up were censored on the last date of known treatment.

In determining the patterns of chemotherapy use at end of life (EOL), only patients who had died and had undergone complete follow-up were included in the analysis. 
Duration of Chemotherapy in Metastatic Breast Cancer

\section{Results}

\section{Patient and Disease Characteristics}

Of the 318 patients with $\mathrm{MBC}$ with complete information on ER, PR, and HER2 status, 199 patients were included for analysis (Figure 1). Of the 119 patients who were excluded, 43 did not have distant metastasis or locally advanced disease treated with palliative intent, 11 patients did not receive at least 1 line of palliative chemotherapy at DFCI, 38 patients did not receive any palliative chemotherapy, 13 patients had another active malignancy, and 14 patients had at least 2 breast primaries. Also excluded were 32 patients with $\mathrm{HR}^{+}$disease who were still receiving hormonal monotherapy with no receipt of palliative chemotherapy. The median age of these patients was 55 years old (range, 35-88). Patients who had previously received hormonal therapy and then proceeded to chemotherapy were included in the analysis. Subgroup distribution was: $\mathrm{HR}^{+}, 48 \%(\mathrm{n}=96)$; TNBC, $22 \%(n=44)$; and HER2 ${ }^{+}, 30 \%(n=59)$. Median follow-up was 2.4 years (range, 0.1-8.6). Median age at metastatic diagnosis was 53 years (range, 28-87).

No major differences in age, race, menopausal status, disease stage, or receipt of adjuvant/neoadjuvant chemotherapy were seen between subtypes (Table 1). As expected, histologic grade $(P<.0001)$, receipt of adjuvant/neoadjuvant endocrine therapy $(P<.0001)$, receipt of adjuvant/neoadjuvant trastuzumab therapy $(P<.0001)$, and disease-free interval $(P<.0001)$ varied between subtypes. Although no significant differences were seen in the number of metastatic sites, statistically significant differences in sites of first and subsequent metastasis were seen between the subtypes. For example, central nervous system (CNS) metastases were noted in half of the patients with either HER2-amplified or TNBC subtypes, compared with $26 \%$ of patients with $\mathrm{HR}^{+}$cancers $(P=.0011)$. Chest wall recurrences were noted in $32 \%$ of patients with TNBC, compared with $9 \%$ and $15 \%$ of patients with $\mathrm{HR}^{+}$or HER2-amplified tumors, respectively $(P=.0048)$. Bone metastases were most common in patients with $\mathrm{HR}^{+}$tumors $(P<.0001)$; liver metastases were more common in patients with $\mathrm{HR}^{+}$or HER2-amplified tumors, compared with patients with TNBC $(P=.0010)$.

\section{Treatment}

The most commonly used chemotherapies in the first-line setting included capecitabine, followed by vinorelbine and paclitaxel. No major qualitative differences were seen in chemotherapy use in the first line, except for the inclusion of anti-HER2 therapy in patients with HER2-amplified disease. A total of 11,21 , and 15 patients were enrolled in clinical trials in the first, second, and third line of treatment, respectively (Appendix B, available online, in this article, at JNCCN.org).

Patients with HER2-amplified MBC received more lines of chemotherapy than patients with other subtypes $(P=.0320)$. In addition, the proportion of patients receiving chemotherapy by line was consistently similar or higher in HER2-amplified tumors compared with other subtypes (Figure 2). For example, nearly $60 \%$ of patients with HER2-amplified tumors were treated with fourth-line chemotherapy, compared with approximately $40 \%$ of patients with $\mathrm{HR}^{+}$or TNBC tumors.

The duration of chemotherapy received by patients also differed by subtype, with a trend for pa-

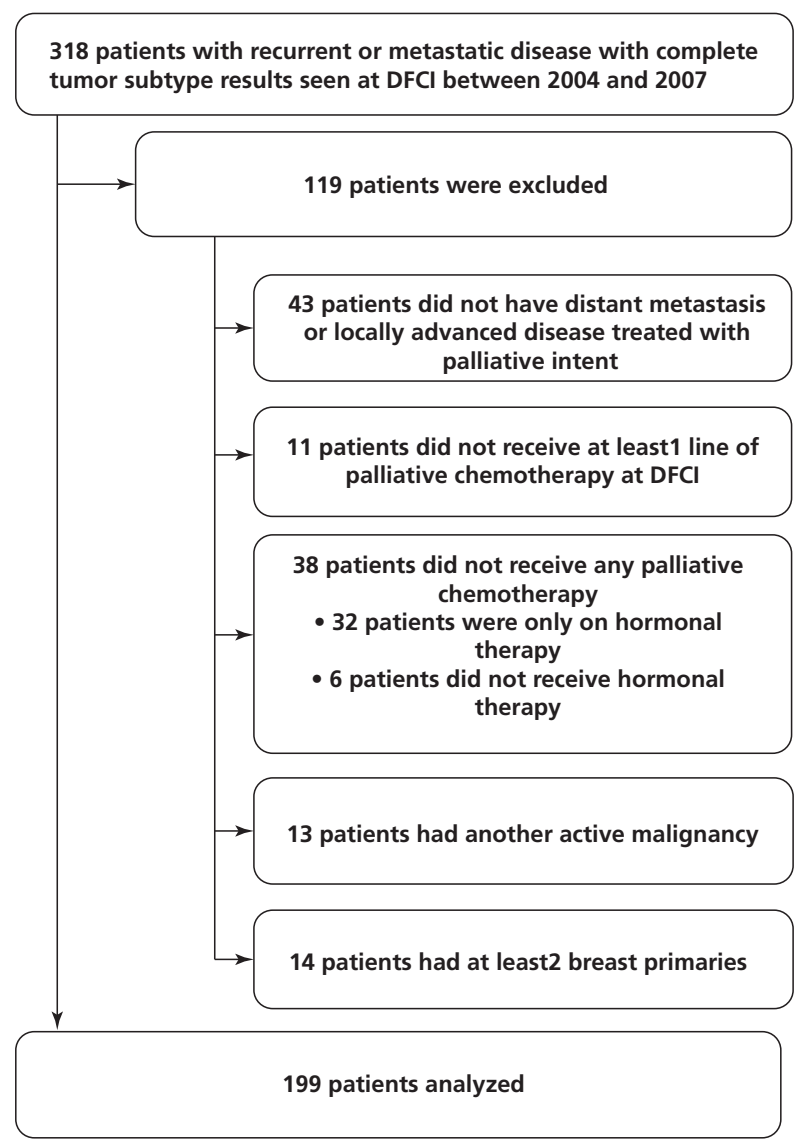

Figure 1 Patients excluded from analysis. Abbreviation: DFCl, Dana-Farber Cancer Institute. 
Seah et al

\begin{tabular}{|c|c|c|c|c|c|}
\hline & Total & $\mathbf{H R}^{+}$ & TNBC & HER2 $^{+}$ & $P$ Value \\
\hline Number of patients (\%) & 199 & $96(48)$ & $44(22)$ & $59(30)$ & \\
\hline \multicolumn{6}{|l|}{ Median Age (range) } \\
\hline Initial diagnosis & $49(26-83)$ & $50(27-81)$ & $48(28-73)$ & $49(26-83)$ & .3692 \\
\hline Metastatic diagnosis & $53(28-87)$ & $54(30-84)$ & $49(28-75)$ & $52(29-87)$ & .2332 \\
\hline \multicolumn{6}{|l|}{ Race (\%) } \\
\hline White & $184(92)$ & $88(92)$ & $40(91)$ & $56(95)$ & .9115 \\
\hline Black & $8(4)$ & $4(4)$ & $2(5)$ & $2(3)$ & \\
\hline Others & $7(4)$ & $4(4)$ & $2(5)$ & $1(2)$ & \\
\hline \multicolumn{6}{|l|}{ Menopausal Status (\%) } \\
\hline Premenopausal & $107(54)$ & $55(57)$ & $21(48)$ & $31(53)$ & .4680 \\
\hline Postmenopausal & $90(45)$ & $41(43)$ & $22(50)$ & $27(46)$ & \\
\hline Unknown & $2(1)$ & $0(0)$ & $1(2)$ & $1(2)$ & \\
\hline \multicolumn{6}{|c|}{ Stage at Initial Presentation (\%) } \\
\hline 1 & $18(9)$ & $8(8)$ & $4(9)$ & $6(10)$ & .0981 \\
\hline 2 & $68(34)$ & $35(37)$ & $14(32)$ & $19(32)$ & \\
\hline 3 & $55(28)$ & $21(22)$ & $20(46)$ & $14(24)$ & \\
\hline 4 & $57(29)$ & $31(32)$ & $6(14)$ & $20(34)$ & \\
\hline Missing & $1(1)$ & $1(1)$ & $0(0)$ & $0(0)$ & \\
\hline \multicolumn{6}{|l|}{ Grade (\%) } \\
\hline 1 & $13(7)$ & $12(13)$ & $0(0)$ & $1(2)$ & $<.0001$ \\
\hline 2 & $62(31)$ & $43(45)$ & $5(11)$ & $14(24)$ & \\
\hline 3 & $118(59)$ & 37 (39) & $38(86)$ & $43(73)$ & \\
\hline Unknown & $6(3)$ & $4(4)$ & $1(2)$ & $1(2)$ & \\
\hline Neoadjuvant Therapy (\%) & $\mathrm{N}=142$ & $\mathrm{n}=65$ & $n=38$ & $\mathrm{n}=39$ & \\
\hline Yes & $37(26)$ & $14(21)$ & $11(29)$ & $12(31)$ & .7466 \\
\hline No & $104(73)$ & $50(77)$ & $27(71)$ & $27(69)$ & \\
\hline Missing & $1(1)$ & $1(2)$ & $0(0)$ & $0(0)$ & \\
\hline \multicolumn{6}{|c|}{ Adjuvant/Neoadjuvant Chemotherapy (\%) } \\
\hline Yes & $122(86)$ & $53(82)$ & $35(92)$ & $34(87)$ & .5890 \\
\hline No & $19(13)$ & $11(17)$ & $3(8)$ & $5(13)$ & \\
\hline Missing & $1(1)$ & $1(2)$ & $0(0)$ & $0(0)$ & \\
\hline \multicolumn{6}{|c|}{ Adjuvant/Neoadjuvant Endocrine Therapy (\%) } \\
\hline Yes & $80(56)$ & $58(89)$ & $0(0)$ & $22(56)$ & $<.0001$ \\
\hline No & $61(43)$ & $6(9)$ & $38(100)$ & $17(44)$ & \\
\hline Missing & $1(1)$ & $1(2)$ & $0(0)$ & $0(0)$ & \\
\hline \multicolumn{6}{|c|}{ Adjuvant/Neoadjuvant Anti-HER2 Therapy (\%) } \\
\hline Yes & $14(10)$ & $0(0)$ & $0(0)$ & $14(36)$ & $<.0001$ \\
\hline No & 127 (89) & $64(100)$ & $38(100)$ & $25(64)$ & \\
\hline Missing & $1(1)$ & $0(0)$ & $0(0)$ & $0(0)$ & \\
\hline
\end{tabular}

Abbreviations: HR, hormone receptor; MBC, metastatic breast cancer; TNBC, triple-negative breast cancer. 
Duration of Chemotherapy in Metastatic Breast Cancer

\begin{tabular}{|c|c|c|c|c|c|}
\hline & Total & $\mathrm{HR}^{+}$ & TNBC & HER2 ${ }^{+}$ & $P$ Value \\
\hline \multicolumn{6}{|l|}{ Median Time Points, mo $(95 \% \mathrm{Cl})$} \\
\hline Disease-free interval (excluding stage IV) & $33(11-103)$ & $46(11-110)$ & $22(6-65)$ & $32(16-104)$ & $<.0001$ \\
\hline Overall survival since metastatic diagnosis & $32(27-41)$ & $36(28-49)$ & $17(10-22)$ & $54(31-66)$ & $<.0001$ \\
\hline \multicolumn{6}{|l|}{ Sites of Metastasis at Initial MBC Diagnosis (\%) } \\
\hline Central nervous system & $9(5)$ & $4(4)$ & $3(7)$ & $2(3)$ & .7430 \\
\hline Liver & $98(49)$ & $47(49)$ & $18(41)$ & $33(56)$ & .3306 \\
\hline Lung & $63(32)$ & $22(23)$ & $20(46)$ & $21(36)$ & .0205 \\
\hline Bone & $116(58)$ & $69(72)$ & $18(41)$ & $29(49)$ & $<.0001$ \\
\hline Lymph node & $119(60)$ & $57(59)$ & $31(70)$ & $31(53)$ & .1880 \\
\hline Chest wall & $20(10)$ & $5(5)$ & $9(20)$ & $6(10)$ & .0275 \\
\hline Others & 78 (39) & $41(43)$ & $16(36)$ & $21(36)$ & .6390 \\
\hline Number of sites at MBC diagnosis (range) & $3(1-6)$ & $3(1-6)$ & $3(1-5)$ & $2(1-5)$ & .6385 \\
\hline \multicolumn{6}{|l|}{ Sites of Metastasis at Last Evaluation (\%) } \\
\hline Central nervous system & 78 (39) & $25(26)$ & $22(50)$ & $31(53)$ & .0011 \\
\hline Liver & $151(76)$ & $81(84)$ & $24(55)$ & $46(78)$ & .0010 \\
\hline Lung & $97(49)$ & $43(45)$ & $26(59)$ & $28(48)$ & .2918 \\
\hline Bone & $140(70)$ & $80(83)$ & $23(52)$ & $37(63)$ & $<.0001$ \\
\hline Lymph node & $140(70)$ & $63(66)$ & $38(86)$ & $39(66)$ & .0247 \\
\hline Chest wall & $32(16)$ & $9(9)$ & $14(32)$ & $9(15)$ & .0048 \\
\hline Others & $131(34)$ & $69(72)$ & $24(55)$ & $38(64)$ & .1360 \\
\hline $\begin{array}{l}\text { Number of sites of metastasis at last } \\
\text { evaluation (range) }\end{array}$ & $4(1-8)$ & $4(1-8)$ & $4(1-8)$ & $4(1-8)$ & .6869 \\
\hline Number of Lines of Chemotherapy (Range) & $3(1-11)$ & $3(1-11)$ & $3(1-8)$ & $4(1-11)$ & .0320 \\
\hline \multicolumn{6}{|l|}{ Vitals (\%) } \\
\hline Dead & $165(83)$ & $82(86)$ & $40(91)$ & $43(73)$ & .0445 \\
\hline Alive or unknown ${ }^{a}$ & $34(17)$ & $14(15)$ & $4(9)$ & $16(27)$ & \\
\hline
\end{tabular}

Abbreviations: $\mathrm{HR}$, hormone receptor; $\mathrm{MBC}$, metastatic breast cancer; $\mathrm{TNBC}$, triple-negative breast cancer.

aVitals were unknown for 1 patient with $\mathrm{HR}+, 1$ patient with TNBC, and 2 patients with HER2 ${ }^{+}$disease.

tients with HER2-amplified tumors receiving longer durations of chemotherapy. The median duration of chemotherapy in patients with HER2-amplified tumors remained at 4 months or more even out to sixthline therapy. Patients with TNBC tended to receive the shortest duration of chemotherapy for every line of therapy (Figure 3). For patients with $\mathrm{HR}^{+}$disease, the median time between $\mathrm{MBC}$ and first chemotherapy was 6.0 months (range, 0-76.9) and the median number of lines of hormonal therapy was 1 (range, 0-3). The proportion of patients with HER2-amplified tumors on chemotherapy for 3 months or more was consistently higher than that of patients with TNBC tumors (Table 2). A cutoff of 3 months was chosen as a proxy for clinical benefit, assuming that most patients would be restaged in this interval. The results were similar using a 6 month cutoff, which would be consistent with the definition of clinical benefit used in many clinical trials (Appendix B, available online, in this article, at JNCCN.org).

\section{Overall Survival}

The median overall survival from metastatic diagnosis for the entire cohort was 32 months. Overall survival time was longest in patients with HER2-amplified disease, followed by $\mathrm{HR}^{+}$and then TNBC disease (54 vs 36 vs 17 months, respectively; $P<.0001$; Table 1 and Figure 4). When patients with HER2amplified disease were further broken down into $\mathrm{HR}^{+}$and $\mathrm{HR}^{-}$disease, the median overall survival time was $53.1(n=31)$ and 55.8 months $(n=28)$, respectively. When the median time between the ini- 
tiation of chemotherapy and death was calculated, the time was 41.3 months for HER2-amplified disease, 18.9 months for $\mathrm{HR}^{+}$disease, and 13.4 months for TNBC.

\section{Chemotherapy at EOL}

At the time of chart review, 165 of the 199 patients had died. Fourteen patients who had died but were lost to follow-up $(n=14)$ were excluded in this analysis. The median length of time between the last date of chemotherapy and death was 37 days. No statistically significant differences were seen between the subtypes $(P=.3280$; Table 3$)$. Of the 151 patients who died and underwent complete follow-up, 27 (18\%) received chemotherapy within the last 14 days of life.

\section{Discussion}

A retrospective review was conducted of patients with $\mathrm{MBC}$ in a single academic institution between 2004 and 2007, describing chemotherapy patterns among patients with 3 common clinical breast cancer subtypes. Patients with HER2-amplified disease were found to receive the most lines and the longest duration of chemotherapy for each line. In contrast, patients with TNBC received fewer lines of chemotherapy, and also tended to have the shortest duration of chemotherapy for each line. Patients with HER2-amplified tumors also experienced the longest overall survival. Finally, the median time from last chemotherapy administration until death was found

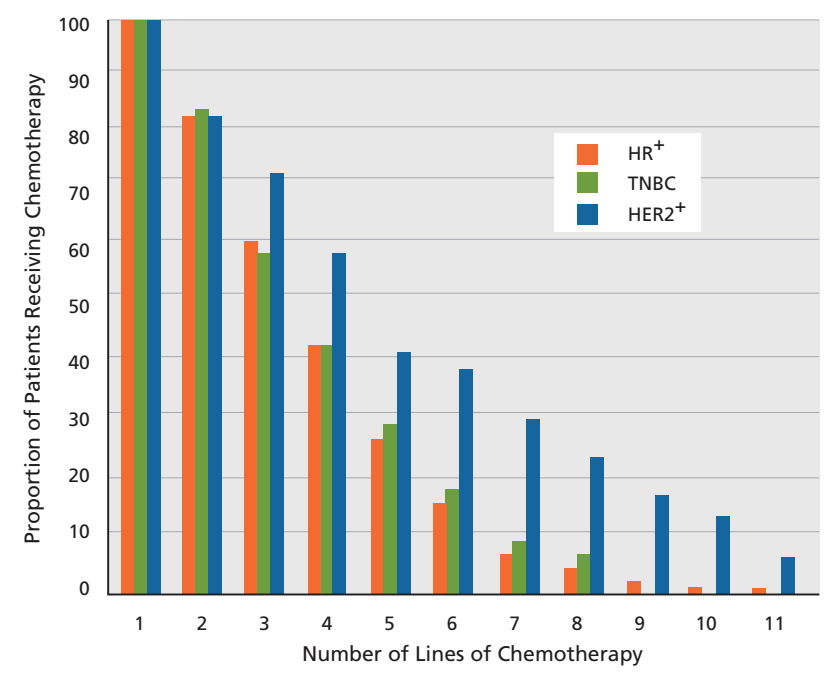

Figure 2 Number of lines of chemotherapy by line and subtype. Abbreviations: HR, hormone receptor; TNBC, triple-negative breast cancer. to be 37 days, with $18 \%$ of patients receiving chemotherapy within the last 14 days of life.

Inadequate evidence currently exists to guide patients and clinicians regarding when to consider later lines of chemotherapy and the likely outcomes of such treatment. The literature suggests that prolonged first-line chemotherapy, and even secondline chemotherapy, is associated with longer overall and progression-free survival. ${ }^{18,24} \mathrm{~A}$ phase III study of eribulin versus treatment of provider choice in the third line and beyond setting showed a 2.5-months survival advantage for eribulin, indicating that later lines of chemotherapy can extend survival in some patients. ${ }^{19}$ In phase II studies of trastuzumab emtansine (TDM1) in highly refractory patients with HER2-amplified MBC (median of 7 lines of palliative chemotherapy), prolonged responses were observed in a high proportion of patients..$^{25}$ Moreover, responses to anti-HER2 therapy can be demonstrated even after progression through TDM1. ${ }^{26}$ On the other hand, the NCCN Clinical Practice Guidelines

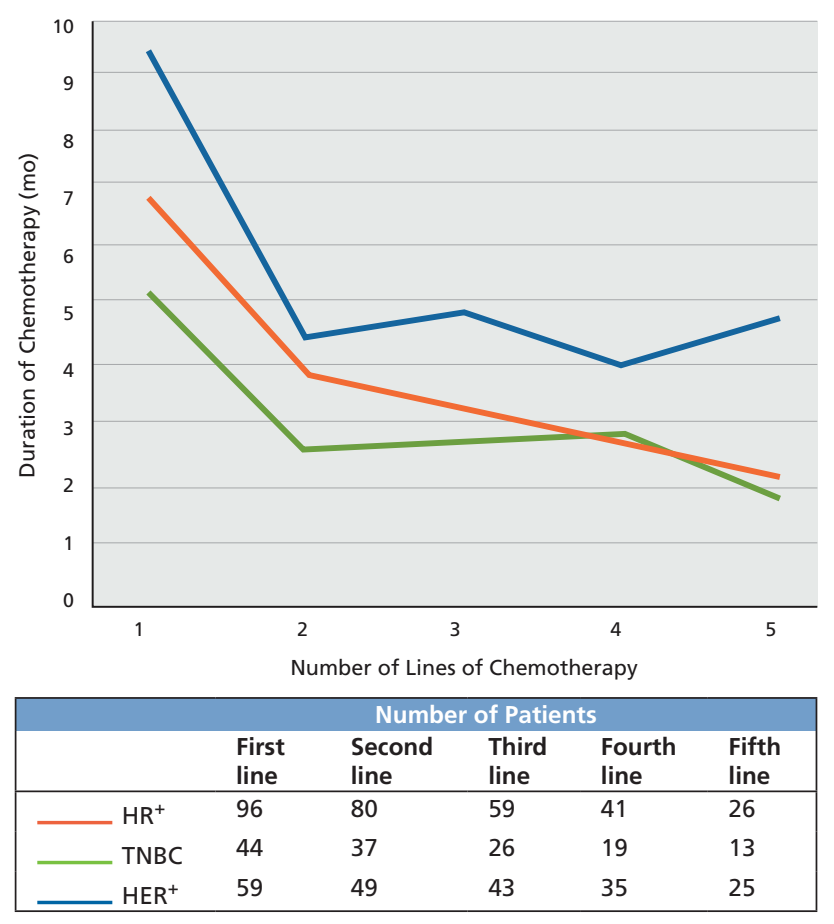

Figure 3 Median duration of chemotherapy according to line and subtype. Only data up to the fifth line of chemotherapy is presented as the number of patients by subtype in the sixth line and beyond is too small ( $<10$ patients in the TNBC category) to draw any conclusions. Of the 33 patients who received first- but not second-line therapy, 14 died after first-line treatment, 7 were lost to follow-up, and 12 had not experienced progression and/or switched to endocrine treatment. Abbreviations: HR, hormone receptor; TNBC, triple-negative breast cancer. 
Duration of Chemotherapy in Metastatic Breast Cancer

Table 2 Proportion of Patients on Chemotherapy for More than 3 Months by Line and Subtype

\begin{tabular}{|c|c|c|c|c|c|c|}
\hline \multirow[b]{2}{*}{$\begin{array}{l}\text { Line of } \\
\text { Chemotherapy }\end{array}$} & \multirow[b]{2}{*}{$\begin{array}{l}\text { Number of } \\
\text { Patients }\end{array}$} & \multicolumn{4}{|c|}{ Number of Patients on Chemotherapy for $\geq 3$ Months } & $P$ Value \\
\hline & & Total & $\mathrm{HR}^{+}$ & TNBC & HER2 $^{+}$ & Fisher Exact Test \\
\hline Third & 128 & $\begin{array}{l}69 \\
(53.9 \%)\end{array}$ & $\begin{array}{l}30 \\
(50.9 \%)\end{array}$ & $\begin{array}{l}10 \\
(38.5 \%)\end{array}$ & $\begin{array}{l}29^{\prime} \\
(67.4 \%)\end{array}$ & .0518 \\
\hline Fourth & 95 & $\begin{array}{l}48 \\
(50.5 \%)\end{array}$ & $\begin{array}{l}18 \\
(43.9 \%)\end{array}$ & $\begin{array}{l}8 \\
(42.1 \%)\end{array}$ & $\begin{array}{l}22 \\
(62.9 \%)\end{array}$ & .2035 \\
\hline Fifth & 64 & $\begin{array}{l}31 \\
(48.4 \%)\end{array}$ & $\begin{array}{l}9 \\
(34.6 \%)\end{array}$ & $\begin{array}{l}3 \\
(23.1 \%)\end{array}$ & $\begin{array}{l}19 \\
(76.0 \%)\end{array}$ & .0016 \\
\hline Sixth & 46 & $\begin{array}{l}26 \\
(56.5 \%)\end{array}$ & $\begin{array}{l}7 \\
(46.7 \%)\end{array}$ & $\begin{array}{l}1 \\
(12.5 \%)\end{array}$ & $\begin{array}{l}18 \\
(78.3 \%)\end{array}$ & .0032 \\
\hline
\end{tabular}

Abbreviations: HR, hormone receptor; TNBC, triple-negative breast cancer.

in Oncology for Breast Cancer ${ }^{27}$ recommend supportive therapy only for patients who do not experience a tumor response to 3 sequential chemotherapy regimens.

In this study, the proportion of patients receiving later lines of chemotherapy declined more rapidly in patients with $\mathrm{HR}^{+}$or TNBC tumors compared with patients with HER2-amplified tumors. In addition, the proportion of patients on chemotherapy for 3 months or more actually increased in patients with HER2-amplified disease with later lines of therapy, but decreased with each line in patients with $\mathrm{HR}^{+}$ and those with TNBC. For example, only 13 of 44 (30\%) of patients with TNBC were treated with fifth-line chemotherapy and, among those treated, only 3 of $13(23 \%)$ remained on therapy for 3 months or more. In contrast, 19 of 25 (76\%) patients with HER2-amplified MBC received fifth-line chemotherapy for at least 3 months. Although the numbers in each line and category are small and should be interpreted with caution, these results suggest that, compared with other subtypes, patients with HER2-amplified disease benefit differentially from later lines of therapy.

Of the limited studies analyzing only patients with $\mathrm{MBC}$, Kennecke et $\mathrm{al}^{28}$ showed that breast cancer molecular subtypes are associated with differences in survival after relapse. The median time from metastasis to death was 2.2 years for luminal A, 1.6 years for luminal B, 1.3 years for luminal/HER2amplified, 0.7 years for HER2-amplified, 0.5 years for basal-like, and 0.9 years for triple-negative nonbasal breast cancers. Brown et $\mathrm{al}^{29}$ found similar results in patients with stage 3-4 disease, with patients with
TNBC having the worst survival, followed by patients with $\mathrm{ER}^{-} / \mathrm{PR}^{-} / \mathrm{HER}^{+}{ }^{+}$disease and other breast cancers (median survival, 2.3 vs 3.6 vs 4.8 years, respectively). Interestingly, in the present study, patients with HER2-amplified disease were found to experience the longest median survival time at 4.5 years, followed by patients with $\mathrm{HR}^{+}$disease at 3.0 years and those with TNBC at 1.4 years $(P<.0001)$. When the HER2-amplified group was further separated according to $\mathrm{HR}$, the median overall survival time was 4.4 years for $\mathrm{HR}^{+}$and 4.7 years for $\mathrm{HR}^{-}$ disease. This discrepancy may be explained by the differences in treatments. The study by Kennecke et $\mathrm{al}^{28}$ predated the widespread use of trastuzumab in HER2-amplified patients. Brown et $\mathrm{al}^{29}$ included patients with stage III disease in their study. The present study included patients who were diagnosed with MBC between 2004 and 2007, when targeted biologic agents such as trastuzumab and lapatinib were widely used. Notably, the present study's survival estimates for patients with $\mathrm{HR}^{+} \mathrm{MBC}$ should be interpreted with some caution, because it excluded patients who did not receive chemotherapy $(n=32)$ but may have been undergoing an extended duration of endocrine therapy and therefore may have experienced better survival outcome. Nevertheless, the present authors believe their results clearly show improvement in survival among patients with HER2amplified MBC compared with historical controls. They also find it notable that these improvements occurred even in the setting of a very high rate of CNS metastasis (53\%).

Finally, the authors found that, despite the expected decrease in the proportion of patients re- 


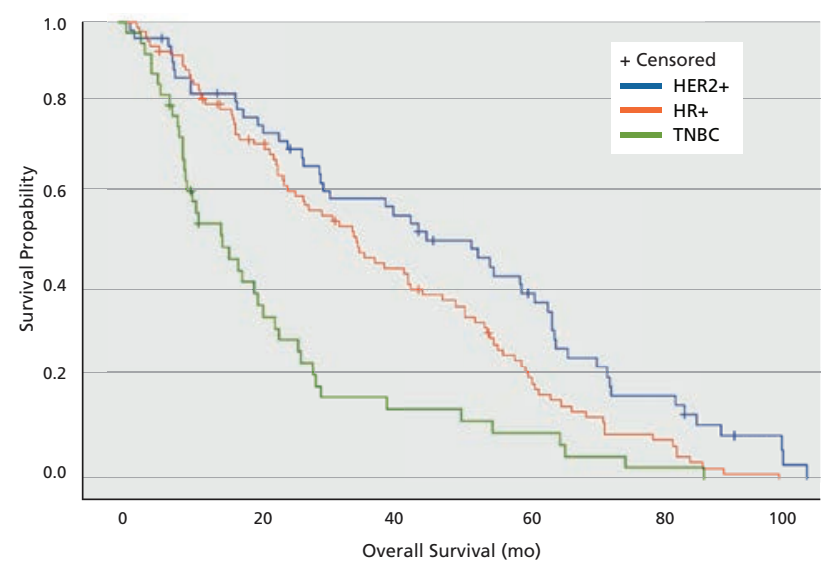

Figure 4 Kaplan-Meier curves for overall survival by subtype from the date of metastatic breast cancer diagnosis.

Abbreviations: HR, hormone receptor; TNBC, triple-negative breast cancer.

ceiving successive lines of chemotherapy, $18 \%$ were treated with chemotherapy within the last 14 days of life. This finding is comparable to that noted in a cohort of 19,000 commercially insured patients with cancer, approximately $17 \%$ of whom were still being treated within 2 weeks of death. ${ }^{30}$

ASCO has created the Quality Oncology Practice Initiative, which has identified chemotherapy cessation in the last 2 weeks of life as one of the benchmarks for improving clinical practice for patients at EOL. In practice, finding the right balance between the appropriate therapy and overly aggressive therapy is challenging. The present authors plan to expand on this work in the future to understand whether there are modifiable factors that could increase the use of hospice and reduce the use of chemotherapy at EOL.

The present study has several limitations. First, it is a relatively small study of patients in a single academic institution, and therefore may not be generalizable to the general community. In an ideal situation, prospectively collected data would be used from large groups of patients treated in both academic and community settings with equal access to treatment options. However, these data are rare. In the absence of this information, retrospective studies provide a pragmatic source of information. Second, the authors did not formally assess the response rates of each line of therapy. They chose not to do this because patients were staged at varying intervals, and clinical radiology reports do not typically adhere to RECIST requirements.
For this reason, the authors used whether the patient was on chemotherapy for 3 or more or 6 or more months as a surrogate marker of clinical benefit. Third, this study only included patients who received chemotherapy. Thus, patients with $\mathrm{HR}^{+}$ tumors who were still on hormonal monotherapy at the time of analysis were excluded, and this could have biased the survival estimates toward a shorter survival. In addition, the authors did not perform a comprehensive analysis of the disease, physician, or patient factors that were associated with use of later lines of chemotherapy. The authors stress that their descriptions of the duration of later lines of chemotherapy are based only on patients who received chemotherapy. Hence, the fact that patients with HER2-amplified MBC in this study were able to remain on later lines chemotherapy for prolonged durations should not be interpreted to mean that all patients with HER2-amplified MBC should receive multiple lines of chemotherapy. Both physicians and patients likely made decisions about chemotherapy use based on multiple factors, including prior response to treatment, toxicities, disease burden, organ function, performance status, and personal preference. Finally, subtypes were based on the assessment provided at the time of diagnosis, and central pathologic review of the tumor samples was not conducted. However, all samples were reviewed for clinical purposes, as is the standard at DFCI. In addition, the authors calculated the number of lines of therapy for patients with HER2 amplified $\mathrm{HR}^{+}$(median, 3 lines of chemotherapy) and HER2-amplified $\mathrm{HR}^{-}$disease (median, 6 lines of chemotherapy). However, the numbers in each of these groups were small $(n=31$ and $n=28$, respectively), and definitive conclusions about the impact of HR status on the number and duration of chemotherapy cannot be drawn.

\section{Implications}

The emergence of targeted therapies has created a divergence regarding what was previously considered a single disease. This and other studies clearly show that the natural history and progression of $\mathrm{MBC}$ differ according to subtypes. Tumor subtype should be taken into consideration when planning additional lines of therapy. Traditionally, the literature supports patients with $\mathrm{MBC}$ to have up to 3 lines of therapy. ${ }^{21}$ The present data show that a substantial proportion of patients with HER2-amplified disease undergo 
Duration of Chemotherapy in Metastatic Breast Cancer

\begin{tabular}{|c|c|c|c|c|c|}
\hline & Total & $\mathrm{HR}^{+}$ & TNBC & HER2+ & $P$ Value \\
\hline Number of patients who died ${ }^{a}$ & 151 & 74 & 38 & 39 & \\
\hline $\begin{array}{l}\text { Median time between last chemotherapy and } \\
\text { death, d (range) }\end{array}$ & $\begin{array}{l}37 \\
(2-1384)\end{array}$ & $\begin{array}{l}37 \\
(2-354)\end{array}$ & $\begin{array}{l}32 \\
(2-385)\end{array}$ & $\begin{array}{l}55 \\
(5-1384)\end{array}$ & .3280 \\
\hline $\begin{array}{l}\text { Number of patients receiving chemotherapy in } \\
\text { the last } 14 \text { days of life }(\%)\end{array}$ & $\begin{array}{l}27 \\
(18)\end{array}$ & $\begin{array}{l}12 \\
(16)\end{array}$ & $\begin{array}{l}7 \\
(18)\end{array}$ & $\begin{array}{l}8 \\
(21)\end{array}$ & .8551 \\
\hline
\end{tabular}

Abbreviations: HR, hormone receptor; TNBC, triple-negative breast cancer.

a Of the 165 patients who died, 14 were lost to follow-up and were not included in this analysis.

therapy for a prolonged period, often receiving fourth or subsequent lines of chemotherapy. Although ongoing HER2-directed therapy after progression is fairly standard in the United States, these results may hold important implications for countries with more restrictive policies on the use of trastuzumab beyond progression. ${ }^{31-33}$

The decision when to stop chemotherapy at $\mathrm{EOL}$ is a complex issue. ${ }^{34-36}$ Many patients undergoing palliative chemotherapy may not comprehend that it is unlikely to be curative, and this may affect their capacity to make informed decisions about when to stop treatment. ${ }^{37}$ Previous groups have attempted to determine stopping rules for chemotherapy for patients with $\mathrm{MBC}$, and to define the limits of treatment. ${ }^{36,37}$ Despite this, chemotherapy continues to be used in patients with advanced cancer. In the present study, no statistically significant differences were seen in time between the last chemotherapy and death among the subtypes. Future studies should be considered to determine factors that may increase the interval between the last chemotherapy and death, and factors that may improve quality of life at EOL.

\section{Conclusions}

This retrospective study showed variability in the number of lines and duration of chemotherapy and overall survival from metastatic diagnosis according to tumor subtype. These findings may have implications on the management of palliative chemotherapy, and affirm the benefits of ongoing HER2-directed therapy beyond first-line palliative chemotherapy.

\section{References}

1. Dawood S, Broglio K, Gonzalez-Angulo AM, et al. Trends in survival over the past two decades among white and black patients with newly diagnosed stage IV breast cancer. J Clin Oncol 2008;26:4891-4898.

2. Gennari A, Conte P, Rosso R, et al. Survival of metastatic breast carcinoma patients over a 20-year period: a retrospective analysis based on individual patient data from six consecutive studies. Cancer 2005;104:1742-1750.

3. Chia SK, Speers CH, D'Yachkova Y, et al. The impact of new chemotherapeutic and hormone agents on survival in a population-based cohort of women with metastatic breast cancer. Cancer 2007;110:973-979.

4. Guarneri V, Conte PF. The curability of breast cancer and the treatment of advanced disease. Eur J Nucl Med Mol Imaging 2004;31(Suppl 1):S149-161.

5. Gennari A, Stockler M, Puntoni M, et al. Duration of chemotherapy for metastatic breast cancer: a systematic review and meta-analysis of randomized clinical trials. J Clin Oncol 2011;29:2144-2149.

6. Perou CM, Sorlie T, Eisen MB, et al. Molecular portraits of human breast tumours. Nature 2000;406:747-752.

7. Cancer Genome Atlas Network. Comprehensive molecular portraits of human breast tumours. Nature 2012;490:61-70.

8. Wang $\mathrm{Y}$, Yin $\mathrm{Q}, \mathrm{Yu} \mathrm{Q}$, et al. A retrospective study of breast cancer subtypes: the risk of relapse and the relations with treatments. Breast Cancer Res Treat 2011;130:489-498.

9. Dent R, Trudeau M, Pritchard KI, et al. Triple-negative breast cancer: clinical features and patterns of recurrence. Clin Cancer Res 2007;13(15 Pt 1):4429-4434.

10. Lin NU, Claus E, Sohl J, et al. Sites of distant recurrence and clinical outcomes in patients with metastatic triple-negative breast cancer: high incidence of central nervous system metastases. Cancer 2008;113:2638-2645.

11. Sanpaolo P, Barbieri V, Genovesi D. Prognostic value of breast cancer subtypes on breast cancer specific survival, distant metastases and local relapse rates in conservatively managed early stage breast cancer: a retrospective clinical study. Eur J Surg Oncol 2011;37:876-882.

12. Slamon DJ, Clark GM, Wong SG, et al. Human breast cancer: correlation of relapse and survival with amplification of the HER2/neu oncogene. Science 1987;235:177-182.

13. Slamon DJ, Leyland-Jones B, Shak S, et al. Use of chemotherapy plus a monoclonal antibody against HER2 for metastatic breast cancer that overexpresses HER2. N Engl J Med 2001;344:783792.

14. Lim E, Metzger-Filho O, Winer EP. The natural history of hormone receptor-positive breast cancer. Oncology (Williston Park) 2012;26:688-694, 696. 
15. Nielsen DL, Kumler I, Palshof JA, et al. Efficacy of HER2-targeted therapy in metastatic breast cancer. Monoclonal antibodies and tyrosine kinase inhibitors. Breast 2013;22:1-12.

16. Early Breast Cancer Trialists' Collaborative Group (EBCTCG), Peto R, Davies C, et al. Comparisons between different polychemotherapy regimens for early breast cancer: meta-analyses of long-term outcome among 100,000 women in 123 randomised trials. Lancet 2012;379:432-444.

17. Bailey CH, Jameson G, Sima C, et al. Progression-free survival decreases with each subsequent therapy in patients presenting for phase I clinical trials. J Cancer 2012;3:7-13.

18. Gennari A, D'Amico M, Corradengo D. Extending the duration of first-line chemotherapy in metastatic breast cancer: a perspective review. Ther Adv Med Oncol 2011;3:229-232.

19. Cortes J, O'Shaughnessy J, Loesch D, et al. Eribulin monotherapy versus treatment of physician's choice in patients with metastatic breast cancer (EMBRACE): a phase 3 open-label randomised study. Lancet 2011;377:914-923.

20. Lin NU, Burstein HJ. EMBRACE, eribulin, and new realities of advanced breast cancer. Lancet 2011;377:878-880.

21. Cardoso F, Di LA, Lohrisch C, et al. Second and subsequent lines of chemotherapy for metastatic breast cancer: what did we learn in the last two decades? Ann Oncol 2002;13:197-207.

22. Seah D, Lin N, Curley C, et al. Informational needs and the quality of life of patients in their first year after metastatic breast cancer diagnosis [abstract]. J Cancer Res 2012;72(Suppl 3):Abstract P6-08-03.

23. Wheler J, Tsimberidou AM, Moulder S, et al. Clinical outcomes of patients with breast cancer in a phase I clinic: the M. D. Anderson cancer center experience. Clin Breast Cancer 2010;10:46-51.

24. Bernardo G, Palumbo R, Bernardo A, et al. Which benefit from subsequent chemotherapy lines beyond the second for women with metastatic breast cancer? Evidence from a single-center retrospective analysis of survivorship [abstract]. J Clin Oncol 2011;29(Suppl 27):Abstract 255.

25. Krop IE, LoRusso P, Miller KD, et al. A phase II study of trastuzumab emtansine in patients with human epidermal growth factor receptor 2-positive metastatic breast cancer who were previously treated with trastuzumab, lapatinib, an anthracycline, a taxane, and capecitabine. J Clin Oncol 2012;30:3234-3241.

26. Olson EM, Lin NU, DiPiro PJ, et al. Responses to subsequent anti-HER2 therapy after treatment with trastuzumab-DM1 in women with HER2-positive metastatic breast cancer. Ann Oncol 2012;23:93-97.

27. Theriault RL, Carlson RW, Allred C, et al. NCCN Clinical Practice Guidelines in Oncology: Breast Cancer. Version 3, 2013. Available at: NCCN.org. Accessed December 9, 2013.

28. Kennecke H, Yerushalmi R, Woods R, et al. Metastatic behavior of breast cancer subtypes. J Clin Oncol 2010;28:3271-3277.

29. Brown BK, Karasavvas N, Beck Z, et al. Monoclonal antibodies to phosphatidylinositol phosphate neutralize human immunodeficiency virus type 1: role of phosphate-binding subsites. J Virol 2007;81:2087-2091.

30. Earle CC, Landrum MB, Souza JM, et al. Aggressiveness of cancer care near the end of life: is it a quality-of-care issue? J Clin Oncol 2008;26:3860-3866.

31. Australian Government Department of Human Services. Late stage metastatic breast cancer. Available at: http://www. medicareaustralia.gov.au/provider/patients/late-breast-cancer.jsp. Accessed August 17, 2012.

32. National Collaborating Centre for Cancer. Advanced breast cancer: diagnosis and treatment. London (UK): National Institute for Health and Clinical Excellence (NICE); 2009. NICE clinical guideline; no. 81 .

33. Pegram M, Liao J. Trastuzumab treatment in multiple lines: current data and future directions. Clin Breast Cancer 2012;12:10-18.

34. Benner SE, Fetting JH, Brenner MH. A stopping rule for standard chemotherapy for metastatic breast cancer: lessons from a survey of Maryland medical oncologists. Cancer Invest 1994;12:451455.

35. Baszanger I. One more chemo or one too many? Defining the limits of treatment and innovation in medical oncology. Soc Sci Med 2012;75:864-872.

36. Harrington SE, Smith TJ. The role of chemotherapy at the end of life: "when is enough, enough?". JAMA 2008;299:2667-2678.

37. Weeks JC, Catalano PJ, Cronin A, et al. Patients' expectations about effects of chemotherapy for advanced cancer. N Engl J Med 2012;367:1616-1625. 